\title{
Factors related to utilization of antenatal care in Nepal: A generalized linear approach
}

\author{
Shrestha G \\ Gauri Shrestha, Reader, Central Department of Statistics, Tribhuvan University, Kirtipur, Nepal.
}

\section{Abstract}

Background: Antenatal care provides an entry point for pregnant women to the health care system. Proper utilization of available health services essential for successful implementation of health programmes.

Objectives: This study investigates factors associated with the use of maternal health services in terms of number of antenatal care visits.

Methods: For analyzing the use of antenatal care health services in Nepal, data was extracted from individual recodes of a data file of Nepal Demographic Health Survey 2006. The unit of analysis for this study was Ever Married Woman (who had at least one live birth in the five years preceding the survey). The sample of study consisted of 4182 Ever Married Woman. The response variable has numbers as its possible outcome therefore generalized Poisson regression model was selected for establishing linkage between number of antenatal care visits and several explanatory variables.

Results: The result of this study shows that women with low education level, those residing in rural areas and those with low socio-economic status are less likely to attend antenatal care visits.

Conclusion: This study confirms that antenatal care visit is indeed an effective entry point for delivery care. Programmes directed at improving and increasing the accessibility of antenatal care are important to improve maternal health, especially in developing countries like Nepal.

Key words: Antenatal care; ever married woman; Poisson regression model

\section{INTRODUCTION}

\begin{abstract}
A ntenatal Care (ANC) is named as one of the four pillars of Safe Motherhood Initiatives'. Routine antenatal visits may raise awareness about the need for care to be taken regarding delivery or make women and their families familiarised with health facilities that enable them to seek help more effectively during a crisis $^{2}$. Other potential benefits of ANC are counseling on the nutrition and healthy pregnancy/delivery behaviour, provide tetanus immunization, malaria prophylaxis, iron and folic acid tablets, screening for anaemia and HIV/AIDS and prevent low birth weight ${ }^{3}$. World Health Organization (WHO) estimates suggest 88 to 98 percent of all pregnancy related deaths are avoidable if all women had access to effective reproductive health services ${ }^{4}$. Therefore antenatal care health service utilization is one of the important factors to reduce the incidence of maternal death. The effectiveness of the health system in
\end{abstract}

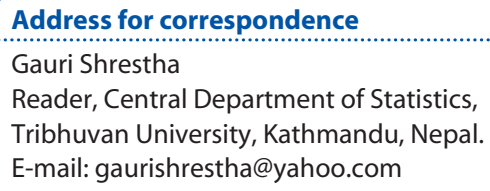

developing countries like Nepal is undermined by lack of utilization of existing facilities. Despite the government's serious commitment to deliver health facility to the door step of common people through innovative approach, the utilization of health services is still far below any acceptable standard. According to a recent data in Nepal, maternal mortality ratio (MMR) is 286 per 100,000 live births ${ }^{5}$. Although MMR appears to have gone down over the past one decade (539 in 1996, 415 in 2000) $)^{6,7}$ it is still high compared to other countries in Asia. Under such circumstances, it becomes vital to identify the factors for non-use of maternal health services.

Coverage of ANC is defined as percentage of women who receive antenatal care at least once during pregnancy for reason related to pregnancy ${ }^{8}$. According to Nepal Demographic Health Survey (NDHS) 2006, three in four (75\%) pregnant women receive antenatal care, about $57 \%$ of mothers who receive antenatal care reported that they were informed about the signs of pregnancy complications ${ }^{5}$. Quality of antenatal care is another issue. Accepted indicators of quality antenatal care include: early initiation, number of ANC visits and medical and educational content of the visits ${ }^{9}$. There has been 
significant improvement over the past ten years in the proportion of mothers who get antenatal care from skilled birth attendant (Doctor, nurse and midwife), increasing from $24 \%$ in 1991 to $28 \%$ in 2001 and $44 \%$ in $2006^{5}$. The Nepal maternal health's program recommends at least four ANC visits: at least one visit in the first trimester, at least one visit in the second trimester and at least two visits in third trimester. Data source shows that only $29 \%$ of mothers meet the recommended schedule (four visits). In the area of maternity care, although women who receive antenatal care are relatively high (75\%) but recommended schedule is still low (29\%). Therefore the present study focuses on the number of ANC visits as indicator of maternal health services.

Utilization of health services is affected not only by access but also by demand for services which is determined largely by socio-economic and demographic factors. Anderson's behavioural model of health service utilization was used as the conceptual frame work $^{10}$. This model has been used extensively in both developed and developing countries, to understand health service utilization ${ }^{11-13}$. The model classified factors that affect health service utilization into three groups: predisposing, enabling and need factors. Predisposing factors are those which present preceding ill health and need for care such as demographic factors, social factors and health beliefs. Among the predisposing factors, the demographic characteristic age reflects the propensity of individuals to use services. Social structure (education, religion) measures the ability of individuals to cope with the problem. Enabling factors provide respondents with the means to make use of the health services. Enabling factors, both personal and organizational must be present for service utilization and these represent the actual ability of the individual to obtain health services. Personal enabling factors include wealth index, Health insurance, regular source of care travel and waiting times. Organizational enabling factors include the availability of health care provider and their spatial distribution ${ }^{10}$. The most immediate cause of health service utilization is the need factor. The judgment about need can be made by the individual himself or family care giver and can be estimated by a self assessment health status, symptoms experience during a period of time. Since all individuals included in the study sample have given live births, the "need" component of Anderson's health seeking behaviour is controlled by sample selection.

This study analyses key variables which past literature identify as determinant of antenatal care. We explore the number of ANC visits varying across different settings. The analysis is based on the generalized linear model with Poisson link function because the response variable, number of ANC visits, has numbers as their possible outcomes.

\section{METHODS}

\section{Data and variables}

For analyzing the number of ANC visits in Nepal, data was extracted from individual recode data file of Nepal Demographic Health Survey (NDHS) 2006. It is a nationally representative survey of 10,793 women of age 15-49 years. The unit of analysis for this study is Ever Married Women (EMW) who had at least one live birth in the five years preceding the survey. For those EMW, who had more than one birth, only utilization behaviour of maternal health services associated with most recent pregnancy within five years was considered. So the sample of this study consists of 4182 EMW.

For this study the maternal health services of interest is the number of antenatal care visits. Therefore number of ANC visit is chosen as dependent variable which has count as its possible outcomes. The predisposing variables included in the model are age, religion and highest level of education. Age is a continuous variable, ranging from 15-49 years. Based on the highest level of education completed, the education is categorised into four levels (no education, primary, secondary and higher). Religion is coded into two categories (Hindu and others). The enabling factors are wealth index, residence, region and working status. Wealth index is the composite variable created from a number of variables and categorized into five levels (poorest, poorer, middle, richer and richest). Residence is categorized into two levels (urban and rural) and region is categorized into five levels (eastern, central, western, mid western and far western).

\section{Statistical Analysis}

In this study, the response variable is number of ANC visits (Y). This variable has counts as its possible outcomes. Generalized linear model with Poisson link function ${ }^{14}$ is particularly useful for response variable that are counts or frequencies and for which it is reasonable to assume an underlying Poisson distribution. Generalized linear model extends ordinary regression model to encompass non normal response distribution and modeling function of the mean. Three components specify a generalized linear model. A random component identifies the response variable y (number of ANC visits) and its probability distribution; a systematic component specifies explanatory variables used in a linear predictor function and a link function specifies the function of 
$\mathrm{E}(\mathrm{y})$ that the model equal to the systematic component. The simplest distribution for a count data is the Poisson. Like counts, Poisson variable can take any non negative integer value. Let y denote a count and let $\mu=E(y)$, the probability function of $y$ is then given by

$f(y)= \begin{cases}\frac{\mu^{y}}{y !} & e^{-\mu}, y=0,1,2 \ldots . . \\ & o, \text { otherwise }\end{cases}$

This has the natural exponential form

$f(y)=a\left(\theta_{i}\right) b\left(y_{i}\right) \exp \left(y_{i} Q\left(\theta_{i}\right)\right)$

Where,

$\theta=\mu, \quad a(\mu)=\exp (-\mu)$

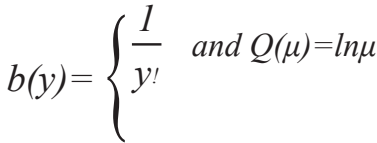

The natural parameter is $\log \mu$. So the link function is the log link $\eta=\log \mu$, the model using this link is

$\ln \mu=\beta_{0}+\sum \beta_{j} X_{i j}$

$\mathrm{i}=1,2, \ldots, \mathrm{n}$

$\mathrm{j}=1,2, \ldots, \mathrm{k}$

This model is called Poisson regression model. The computer package STATA 10.0 has been used for the analysis.

\section{RESULTS}

The frequency and percentage distribution of the number of ANC visits is presented in table $1.28 \%$ have no ANC visits. $9 \%$ have one ANC visits and $19 \%$ have three ANC visits.

The observed and poisson predicted probability are shown in Figure1. It shows that the fitted Poisson distribution under predict 0 's and over predict counts $1,2,3$ and 4

Table 2 shows the mean number of ANC visits by selected background characteristics. Number of ANC visits is remarkably varying by the education level of the women. The women, who had no education have an average 1.67 ANC visits whereas it is 6.26 for the women who have secondary education and it is tripled among the women who have higher education than no education. The number of ANC visits also varies by wealth index. Women who are poorest have an average 1.3 ANC visits where as it is 4.43 for women who are richest. Richest have four times more mean number of ANC visits as compared to poorest. Table 2 also demonstrates that region Midwestern and far western have less mean number of ANC visits compared to eastern, central and western regions.

The $p$ value in table 3 shows that most of the explanatory variables are highly significant. Religion and current working status do not show significant results. The fitted model shows negative association with age and number of children in last five years, residence and region while rest of the variables show positive association. Increase in one year, decreases the expected number of ANC visits by a factor of 0.98 , holding all other variables constant. For every addition of a child, decrease the expected number of ANC visits by $8.4 \%$ holding all other variables constant.

Respondents having higher education increase the expected number of ANC visits by a 2.25 times more likely compared to no education. The estimated coefficients show increasing trend with the wealth index of the respondent.

Tablel 1: Frequency distribution of number of ANC visits:

\begin{tabular}{|ccc|}
\hline No. of ANC visits & Frequency & Percentage \\
\hline 0 & 1161 & 27.76 \\
\hline 1 & 355 & 8.49 \\
\hline 2 & 658 & 15.73 \\
\hline 3 & 797 & 19.06 \\
\hline 4 & 542 & 12.96 \\
\hline 5 & 274 & 6.55 \\
\hline 6 & 178 & 4.26 \\
\hline 7 & 92 & 2.20 \\
\hline 8 & 60 & 1.43 \\
\hline 9 & 27 & 0.65 \\
\hline 10 & 15 & 0.35 \\
\hline 11 & 5 & 0.12 \\
\hline 12 & 10 & 0.24 \\
\hline 13 & 3 & 0.07 \\
\hline 14 & 1 & 0.02 \\
\hline 15 & 3 & 0.07 \\
\hline 19 & 1 & 0.02 \\
\hline Total & 4182 & 100 \\
\hline
\end{tabular}


Table 2: Mean number of ANC visits by selected background characteristics:

\begin{tabular}{lc} 
Variables & $\begin{array}{c}\text { Mean number of } \\
\text { ANC visits }\end{array}$ \\
\hline Predisposing factors: & \\
1) Highest education level: & \\
No education & 1.67 \\
Primary & 2.83 \\
Secondary & 3.87 \\
Higher & 6.26 \\
2) Religion: & \\
Hindu & 2.12 \\
Others & 2.42 \\
\hline Enabling factors: & \\
1) Wealth Index: & \\
Poorest & 1.30 \\
Poorer & 2.03 \\
Middle & 2.49 \\
Richer & 3.06 \\
Richest & 4.43 \\
2) Region: & \\
Eastern & 2.60 \\
Central & 2.70 \\
Western & 2.61 \\
Mid-western & 2.18 \\
Far-western & 1.82 \\
3) Residence: & \\
Rural & 2.78 \\
Urban & 3.58 \\
4) Current working status: & \\
yes & \\
No & 3.18 \\
\hline
\end{tabular}

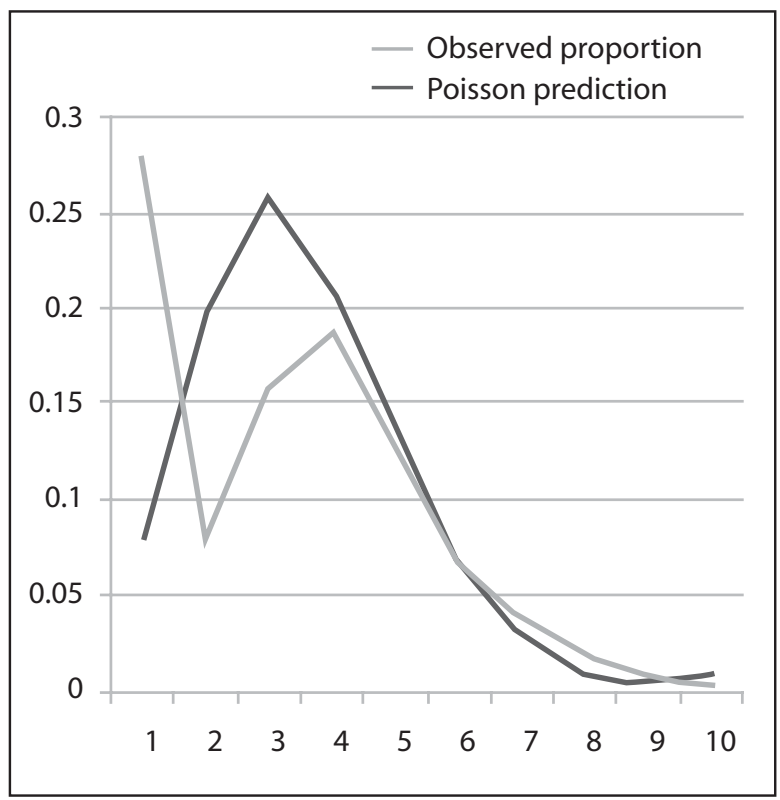

Figure 1: Observed and poisson predicted probability.

\section{DISCUSSION}

There is no doubt that use of maternal health services improve reproductive outcomes ${ }^{15}$. It is well recognized that women's current age play important role in the utilization of maternal health services ${ }^{16}$. In this study, the older women had less number of ANC visits. It may be due to the improvement of education opportunities for women in recent years, younger women might have an enhanced knowledge of modern health care services. Several studies found that physical proximity of health care services; especially in the developing countries play an important role in utilization of health service ${ }^{17}$. This study shows that residence in rural areas use less number of ANC visits. Result of analysis reveal that among the explanatory variables, education and wealth index is the most significant determinant for increasing utilization of number of ANC visits. Several studies have found a strong association between education and utilization of maternal health services ${ }^{18}$. It is found that mother's education is the most consistent and important determinant of use of child and maternal health services. The result from this study also supports the positive association between the education and number of ANC visits. But in rural areas, it does not seem to have greatly improved in number of ANC visits as increase in education level. Women with higher wealth index more often have resources and ability to buy health services. It is obvious from our study that as the wealth index becomes high, number of ANC visits is also increased in both urban and rural areas ${ }^{19}$.This study identified the factors which affect the number of ANC visits as maternal health services utilization and it may be helpful for policy maker/service provider regarding maternal health to make future plans or programme for maternal health in Nepal.

\section{CONCLUSION}

The study confirms that antenatal care visit is indeed an entry point for delivery care. The results indicate that large percentages of women do not seek four or more ANC visits as recommended by World Health Organization (WHO). It is important that programme aimed at improving maternal health include targeting women, especially those from rural areas, with low level education, higher birth order and from poor household given their high risk during pregnancy. This study identified the factors which affect the ANC visits as maternal health services utilization and it may be helpful for policy maker/ service provider regarding maternal health to make future plans or programs for maternal health in Nepal. 
Table 3: Estimation of parameters ofe Poisson regression model for number of ANC visits:

\begin{tabular}{|c|c|c|c|c|c|}
\hline $\begin{array}{l}\text { Variables } \\
\text { Constant }\end{array}$ & $\begin{array}{c}b \\
1.176\end{array}$ & $\begin{array}{l}\text { S.e } \\
.089\end{array}$ & $\begin{array}{l}\text { P-value } \\
<0.0001\end{array}$ & $\begin{array}{c}\mathrm{e}^{\mathrm{b}} \text { (odd ratio) } \\
-\end{array}$ & $\begin{array}{l}\% \\
-\end{array}$ \\
\hline \multicolumn{6}{|l|}{ Predisposing factors: } \\
\hline Age & -0.019 & 0.002 & $<0.0001$ & 0.981 & -1.9 \\
\hline \# of children in last five years & -0.088 & 0.019 & $<0.0001$ & 0.916 & -8.4 \\
\hline \multicolumn{6}{|l|}{$\begin{array}{l}\text { Highest education level: } \\
\text { No education }(R)\end{array}$} \\
\hline Primary & 0.281 & 0.028 & $<0.0001$ & 1.324 & 32.4 \\
\hline Secondary & 0.431 & 0.027 & $<0.0001$ & 1.539 & 53.9 \\
\hline Higher & 0.812 & 0.044 & $<0.0001$ & 2.251 & 125.1 \\
\hline \multicolumn{6}{|l|}{ Religion: } \\
\hline \multicolumn{6}{|l|}{ Hindu (R) } \\
\hline Others & -0.008 & 0.031 & 0.785 & 0.912 & -0.8 \\
\hline \multicolumn{6}{|l|}{ Enabling factors: } \\
\hline \multicolumn{6}{|l|}{ Wealth Index: } \\
\hline \multicolumn{6}{|l|}{ Poorest (R) } \\
\hline Poorer & 0.443 & 0.036 & $<0.0001$ & 1.558 & 55.7 \\
\hline Middle & 0.557 & 0.036 & $<0.0001$ & 1.746 & 74.6 \\
\hline Richer & 0.661 & 0.041 & $<0.0001$ & 1.937 & 93.7 \\
\hline Richest & 0.814 & 0.089 & $<0.0001$ & 2.258 & 125.8 \\
\hline \multicolumn{6}{|l|}{ Region: } \\
\hline \multicolumn{6}{|l|}{ Eastern(R) } \\
\hline Central & -0.125 & 0.032 & $<0.0001$ & 0.883 & -11.7 \\
\hline Western & -0.128 & 0.032 & $<0.0001$ & 0879 & -12.1 \\
\hline Mid-western & -0.273 & 0.034 & $<0.0001$ & 0.761 & -23.9 \\
\hline Far-western(R) & -0.208 & 0.035 & $<0.0001$ & 0.812 & -18.8 \\
\hline \multicolumn{6}{|l|}{ Residence: } \\
\hline Rural & -0.092 & 0.024 & $<0.0001$ & 0.912 & -8.8 \\
\hline \multicolumn{6}{|l|}{ Urban (R) } \\
\hline \multicolumn{6}{|l|}{$\begin{array}{l}\text { Current working status: } \\
\text { yes }(R)\end{array}$} \\
\hline No & -0.031 & 0.023 & 0.168 & 0.969 & -3.1 \\
\hline
\end{tabular}

\section{ACKNOWLEDGEMENTS}

I acknowledge gratefully the guidance and encouragement of Prof. Dr. Ganga Shrestha, Tribhuvan University, Kirtipur. It is my pleasure to acknowledge INSA-NAST bilateral exchange program for funding research at Centre for Mathematical Sciences Kerala, India (Nov 2010-April 2011) and University Grant Committee, Sanothimi, Bhaktpur for the financial support to carry out this doctoral research work.

\section{REFERENCES}

1. World Health Organization. Mother-Baby Package: Implementing safe motherhood in countries, practical guide. Document WHO/FHE/MSM/94.11. Geneva1994.

2. Palaniappan B. Roll of antenatal care in safe motherhood. J Indian Med Assoc. 1995:93:52-4.

3. United Nations Population Fund. Maternal and Neonatal Health in East and South East Asia, Bangkok. 2006.

4. Kunst $A E$, Houweling $T$. A global picture of poor rich differences in the utilization of delivery care in safe motherhood strategies: A review of the evidence. ITG Press, Belgium. 2001;17:297315.
5. Ministry of Health and Population (MOPH) [Nepal], New ERA, and Macro International Inc. 2007. Nepal Demographic Health Survey 2006. Kathmandu, Nepal: Ministry of Health and Population, New ERA and Macro International Inc; 2007.

6. WHO, UNICEF. Estimation of Maternal Mortality, A new approach by WHO and UNICEF.1996.

7. National Planning commission (NPC), The tenth plan. Kathmandu, Nepal: National Planning Commission; 2002.

8. World Health Organization. Coverage of prenatal care: a listing of available information, fourth edition,(WHO/FRH/MSM 196.28). Geneva: WHO; 1996. 
9. Sanders-Philips K, Davis S. Improving prenatal care services for low income African Americanwomen and infants. J Healthcare Poor Underserved. 1998;9(1):14-29.

10. Anderson RM. Revisiting the behavioral model and access to medical care: Does it matter? J Health Social Behav. 1995;36(1):1-10.

11. Forbes DA, Janzen BL. Comparison of rural and urban users and non users of homecare in Canada. Can J Rural Med. 2004;9(4):227-35.

12. Sunil TS, Rajaram S, Zottareli LK. Do individual and program factors matter in the utilization of maternal care services in rural India? a theoretical approach. Social Sci Med. 2006; 62(8):1943-57.

13. Trinh LT, Dibley MJ, Byles J. Determinant of antenatal care utilization in three rural areas of Vietnam. Public Health Nurse. 2007;24(4):300-10.

14. McCullagh $P$, Nelder JA. Generalized linear model, 2nd ed. Chapman \& Hall Inc: NewYork; 1989.
15. Letamo G, Majelantle RG. Factors influencing low birth weight and prematurity in Botswana. J Biosocial Science. 2001;33:391-403.

16. Fosu GB. Childhood morbidity and health care utilization: Cross national comparison of user related factors from DHS data. Social Science Med. 1994;38:1209-20.

17. Paul BK. Health service resources as determinant of infant death of rural Bangladesh, an empirical study. Social Science Med. 32:43-9.

18. Becker S, Peters DH, Gray RH, Ggultiano C, Blake RE. The determinant of use of maternal child health services in Metro Cebu, the Philippines. Health Transition Rev. 1993;3:77-89.

19. Shen C, Williamson JB. Maternal mortality, women's status and economic dependency in less developed countries: cross national analysis. Social Science Med. 49:197-214. 